\title{
DE LAS VIDAS EJEMPLARES A LAS BIOGRAFÍAS COLECTIVAS DE LOS MÉDICOS. UNA PERSPECTIVA CRÍTICA
}

\author{
Guillermo Olagüe de Ros \\ Catedrático de Historia de la Ciencia. Departamento de Anatomía Patológica e \\ Historia de la Ciencia. Facultad de Medicina. Granada
}

\begin{abstract}
RESUMEN
La biografía es un modo de hacer historia de la medicina que cuenta con una larga tradición. Es, quizás, uno de los métodos más clásicos y reconocidos. Aunque aparentemente pueda parecer fácil y sencillo, si se realiza con seriedad y rigor es francamente difícil. Diversos condicionantes de tipo psicológico — especialmente, la identificación del historiador con el biografiado- pueden convertir una biografía en una caricatura carente de interés y proyección. Los acercamientos provincianos y el énfasis en el precursorismo pueden contribuir también a deslucir este método. Más reciente es la prosopografía, o estudio biográfico de una colectividad afín. En la prosopografía es más difícil que se den los inconvenientes antes señalados, pero es un acercamiento más laborioso y exigente. Una y otro pueden ser de gran utilidad como apoyo en la enseñanza de la historia de la medicina, especialmente si se tiene en cuenta el parco conocimiento médico con que los alumnos llegan a nuestra disciplina en el momento presente.
\end{abstract}

PALABRAS CLAVE: Biografía, prosopografía, métodos de historia de la medicina, enseñanza de la historia de la medicina

\section{SUMMARY}

As a historical method biography has a long tradition in the studies of the history of medicine. Is perhaps one of the most classical and used methods, but is very difficult when is applied seriously. Many problems, especially form a psychological point of view -identity of the historian with the subject of the biography - can do the results of this approach be without interest. Parochialism and precursorism are circumstances that the historian must avoid too. Historically prosopography, the study of a collectivity of scientists, is a more recent method.. Apparently is more objective, but required more effort . Both, biography and prosopography, can be very useful in the teaching of the history of medicine at present moment, specially when students have a very little knowledge of the greats paradigms of the present medicine.

KEY WORDS: Biography, prosopography, methods in the history of medicine, teaching of the history of medicine 


\section{INTRODUCCIÓN}

Este trabajo tiene por objetivo mostrar desde una perspectiva crítica el posible uso y recurso de las biografías médicas en la docencia de la historia de la medicina. El actual estudiante de medicina se enfrenta a nuestra disciplina, por término general, en el período preclínico, cuando todavía carece de una imagen adecuada de los grandes paradigmas de la medicina, tanto desde el punto de vista de la salud como de la enfermedad. Parece ser, pues, que este modo de hacer historia de la medicina puede ser idóneo y atractivo a tenor de las carencias de todo orden que presenta este alumnado. Bien es verdad que nunca este modelo historiográfico ha estado marginado de la enseñanza médica. Con mayor o menor amplitud e intensidad todas las unidades docentes de historia de la medicina de las Facultades de Medicina españolas lo han utilizado en sus lecciones teóricas o en sus clases practicas, especialmente cuando se ha pretendido iniciar al alumno en el análisis de textos clásicos. Sin embargo, lo que pretendo analizar en este artículo es de la conveniencia o no de reforzar el componente biográfico en la enseñanza de nuestra disciplina, ya sea el individual o el colectivo, dado el derrotero de los actuales planes de estudio.

\section{BIOGRAFÍAS INDIVIDUALES: DE LA CRÍTICA NECESARIA A LA SANTIFICACIÓN LAICA}

En el género narrativo biográfico juega un papel primordial la función, es decir, la intención del narrador hacia sus lectores al escribir la biografía de un personaje concreto. Por término general puede afirmarse que desde este punto de vista las biografías de personajes célebres de la historia de la medicina o de la ciencia no han cumplido, salvo excepciones, un fin pedagógico, pues han sido utilizadas como ejemplo beatífico del genio creador. Es decir, se ha considerado al biografiado como un modelo a seguir, obviándose, en ocasiones por discordantes con la intencionalidad de la narración, aquellos aspectos de su vida, incluso científica, que podían romper la imagen excepcional del sujeto estudiado. Esta tendencia a transformar las vidas de los científicos en hagiografías o vidas ejemplares explica en parte que durante mucho tiempo este modo literario fuera denostado y considerado menor, más propio de aficionados que de rigurosos historiadores y que, desde una perspectiva docente, cumplieran escasamente un fin formativo.

Dos estudios relativamente recientes sobre la misma figura, Andrés Vesalio, me parecen paradigmáticos en cuanto a rigurosidad en el enfoque, pero 
discrepantes en cuanto a las conclusiones. El primero de ellos se debe a José Barón Fernández, pediatra valenciano y perspicaz investigador, que en 1970 publico una monografía con el titulo Andrés Vesalio. Su vida y su obra ${ }^{1}$. Aunque el título es bastante aséptico Barón toma claro partido por el profesor patavino y concluye que Vesalio fue uno de los frutos cimeros del Renacimiento. Toda la obra, pues, está dedicada a mitificar los logros de Vesalio, con una gran rigurosidad y cuidadoso manejo de las fuentes. En 1994 Juan Barcia Goyanes, anatomista de dilatada carrera, editaba en Valencia El mito de Vesalio ${ }^{2}$, título francamente comprometido en contraste con el de Barón, un concienzudo estudio dirigido a desmontar algunos de los logros anatómicos atribuidos a Vesalio. Para Barcia la mitificación del anatomista belga se inició durante la Ilustración $\mathrm{y}$, hasta prácticamente finales del siglo XX, se había mantenido incólume su figura. A revisar el mito dedica Barcia esta monografía, escrita de manera muy atractiva y documentada. Aun siendo dispares en sus conclusiones estas dos obras, por la seriedad en el abordaje del problema, constituyen dos hitos de la historiografía médica publicada en España. Que las conclusiones a las que llegan estos dos estudiosos sean dispares carece de importancia. Lo que interesa es cómo han comprendido a Vesalio en su tiempo, qué análisis han realizado de su obra, y como han ensamblado las distintas biografías del autor con el objeto de explicar genéticamente la evolución de su pensamiento, desde su etapa juvenil hasta su madurez. Tanto Barón como Barcia responden de forma hartamente satisfactoria estas y otras cuestiones.

Como contraste, y fuera del ámbito de la historia de la medicina, daré cuenta de dos estudios biográficos, de parca calidad científica, que representan, a mi modo de entender, dos claras expresiones de lo que podríamos denominar vidas ejemplares o hagiografias históricas. En el año 2000 Esperanza Martínez Montalvo publicaba un libro dedicado a Javier Lasso de la Vega, documentalista a quien se debe la implantación de la Clasificación Decimal Universal en la Biblioteca Nacional de Madrid³. Al descontextualizar al autor en su tiempo, Martínez Montalvo hace de Lasso de la Vega el creador de esta disciplina en España, olvidándose de los ricos antecedentes y de la importante y meritoria labor, entre otros, de Jordi Rubió i Balaguer en la Mancomunitat

1 BArón Fernández, J. (1970), Andrés Vesalio. Su vida y su obra. Madrid, Consejo Superior de Investigaciones Científicas.

2 Barcia, J. (1994), El mito de Vesalio. Valencia, Real Academia de Medicina de la Comunidad Valenciana-Universitat de València, $241 \mathrm{pp}$.

3 Martínez Montalvo, E. (2000), Investigación y producción científica en Documentación: La obra de Javier Lasso de la Vega (1892-1990). Madrid, Editorial Fragua, 366 pp. 
de Catalunya. Todo lo más recuerda la autora como antecedente el hecho de que Lasso pertenecía a una saga familiar de médicos con intereses librescos que se remontaba hasta finales del siglo XVIII. Este trabajo representa, pues, el típico ejemplo de adanismo científico. Es decir, se obvia lo previo y evidente con el fin de glorificar el sujeto estudiado. El segundo estudio se debe a Susana y Virginia Ramírez Martín, quienes en Raíces publicaron en 2004 una nota sobre Joseph Heydeck, personaje curioso que quiso aprovechar el ímpetu de la vacuna jenneriana en España para su propio beneficio al anunciar que había descubierto en cabras de Madrid unas pústulas variolosas iguales que las que padecían las vacas inglesas ${ }^{4}$. El título del artículo «Juan José Heydeck. Un humanista en busca de la Salud Pública» es de por sí bastante expresivo. La tesis que sostienen estas autoras es que Heydeck fue un riguroso científico, si bien era un ex-rabino convertido al catolicismo, un incomprendido, envidiado y vituperado por lo más granado de la profesión médica española de principios del siglo XIX, que le impidió arteramente difundir sus hallazgos ${ }^{5}$.

¿Cuáles son las causas de que en el binomio Barón/Barcia, desde una gran rigurosidad metodológica, los resultados sean tan diferentes, y de que en los acercamientos de Martínez y Ramírez - menos rigurosos - se concluya elevando a los altares a sus respectivos biografiados?. El propio devenir histórico del género biográfico puede ayudar a contestar a estas cuestiones.

Como es sobradamente conocido, el género biográfico cuenta con una dilatada tradición en la medicina occidental. Hasta bien entrado el siglo XVIII tal modo narrativo respondió a diversos motivos. El de más peso, como forma de justificación de la nobleza de la profesión médica. Al glorificar a determinados médicos se salvaguardaba al conjunto de la profesión de ataques infundados. De esta forma el médico quedaba reforzado socialmente. Pero también había una razón de utilidad. Al conocer lo que los médicos precedentes habían creado, podía construirse el saber y la práctica médicas contemporáneas de manera más sólida. No debe olvidarse además que desde la Antigüedad Clásica hasta bien entrado el siglo XIX los médicos no tuvieron conciencia de la evolución progresiva de sus saberes. Todos, aunque hubieran desaparecido hacía siglos, eran contemporáneos desde el punto de vista doctrinal. La medicina medieval árabe enriqueció este género biográfico con una cuidadosa

4 Acerca de Heydeck puede consultarse, como contraste: OlagüE DE Ros, G.; ASTRAIN Gallart, M. (2004), (Salvad a los niños!: los primeros pasos de la vacunación antivariólica en España (1799-1805). Asclepio, 56 (1), 7-31.

5 Ramírez Martín, S.M.; RAmírez Martín, V. (2004), Juan José Heydeck. Un humanista en busca de la Salud Pública. Raíces. Revista Judia de Cultura, 18 (58), 59-65. 
bibliografía de los autores estudiados, fenómeno que en la medicina occidental se dio a partir del Renacimiento. De este período son dignas de mención obras con títulos tan significativos como el Liber de Medicinae Claris Scriptoribus (1506) de Symphorien Champier (1472-ca. 1535) y el Catalogus Illustrium Medicorum (1530) de Otto Brunfels (1488-1534). Con los términos ilustres y notables escritores médicos, su autores tomaban claro partido por la glorificación de los médicos biografiados y como recurso de defensa de la profesión medica. Más atenta a las bibliografías de los médicos es la Bibliotheca Universalis de Conrad Gessner (1545-1555), género que alcanza un importante nivel de erudición en la celebérrima Bibliotheca Scriptorum Medicorum Veterum et Recentiorum (1731) de Jean Jacques Manget (1652-1742), obra en la que confluyen la tradición biográfica con la erudición bibliográfica de la época. Las bibliotecas de Albrecht von Haller (1708-1777) representan, sin duda, la culminación de este género bibliográfico ${ }^{6}$. Es, precisamente, a fines del siglo XVII cuando los modos biográfico y bibliográfico empiezan a convertirse en acercamientos históricos gracias a las nuevas técnicas de erudición. La historia pragmática de la medicina (1792-1803) de Kurt Sprengel (1766-1833) constituye un hito en este sentido. El impacto del positivismo a mediados del siglo XIX supuso la ruptura entre el saber del pasado y el presente. La idea de obsolescencia de las doctrinas y teorías hizo que todo lo que no era contemporáneo, por tanto vivo, estaba periclitado. Ello no significó la desaparición de la historia de la medicina.

Con figuras como Heinrich Haeser (1811-1884) nació una auténtica ciencia de la historia de la medicina. La historia médica exigía técnicas rigurosas y una sólida formación por sus cultivadores. El triunfo de la mentalidad positiva supuso un fuerte respaldo al género biográfico. Para los positivistas, como Carl August Wunderlich (1815-1877), con diferencias notables en algunos aspectos con Haeser, la biografía de los grandes médicos era un método idóneo para conocer la evolución progresiva del pensamiento creador. Los grandes médicos, pues, ha aportando a la humanidad bienestar y mejoras para

6 Bibliotheca Botanica Qua Scripta ad rem herbariam facientia a rerum initiis recensentur. 2 vols., Tiguri, apud Orell, Gessner, Fuessli, et Soc. (1771-1772); Bibliotheca Chirurgica. Qua scripta ad artem chirurgicam facientia a rerum initiis recensentur. 2 vols., Bernae et Basileae, Haller et Scweighauser (1774-1775); Bibliotheca Anatomica. Qua scripta ad anatomem et physiologiam facientia a rerum initiis recensentur. 2 vols., Tiguri, apud Orell, Gessner, Fuessli et Soc., (1774-1777); Biblioteheca Medicinae Practicae qua scripta ad partem medicinae practicam facientia a rerum initiis ad a. 1775 recensentur. 4 vols., Basileae, Joh. Scheighauser; Bernae apud Em. Haller (1776-1778). 
sus condiciones de vida. Un científico excepcional es un individuo excepcional en todos los órdenes de su vida y, por ello, está exento de crítica. Todo lo más, los elementos de su vida que pudieran ser discordantes con esta visión no empañaban un ápice la imagen hagiográfica que el estudioso podía hacer de su biografiado. Las ideas presentes son el resultado de una evolución a partir de doctrinas expuestas en períodos anteriores por mentes geniales. Si queremos conocer bien nuestro presente, pues, hemos de estudiar a esos autores precedentes. Pero en la historia de la medicina existen periodos brillantes, con figuras revolucionarias, y épocas mudas, en las que apenas se produjo adelanto científico. Los prehipocráticos, Salerno y la Edad Media, entre otros momentos, se corresponderían con esas fases de oscuridad científica, mientras que el Renacimiento, con figuras de la talla de Andrés Vesalio, sería una época de gran fertilidad y avance en el conocimiento. Con todo, bien es cierto que desde la mentalidad positiva se consiguieron biografías de médicos francamente notables, pues la rigurosidad en el análisis de las ideas y una adecuada contextualización en el tiempo fueron la mejor garantía para un buen hacer histórico. Esta necesidad de conocer los precedentes explica que algunos de los grandes clínicos de la época se sintieran atraídos por analizar en profundidad las grandes figuras del pasado. No se olvide que Rudolf Virchow (18211902), por ejemplo, dedicó algunos notables estudios a patólogos previos, como Francis Glisson (1597-1677) y Giambattista Morgagni (1682-1771), entre otros. Sin embargo, esa imagen progresista lineal del primer positivismo historiográfico fue matizada de manera importante por Karl Sudhoff (18531938) que rompió, entre otras cosas, con la vieja imagen de los períodos oscuros y las etapas fértiles de la historiografía positivista más radical. Como es bien sabido, Sudhoff fue el iniciador de una sólida línea de trabajo en historia de la medicina de la Edad Media.

Henry E. Sigerist (1891-1957), sucesor de Sudhoff en el instituto de Leipzig desde 1925, acabó definitivamente con la imagen del presente como culminación progresista del pasado. A Sigerist debemos Los grandes Médicos. Historia Biográfica de la Medicina, un magnífico exponente de biografías de médicos notables elaboradas de forma amena pero con gran rigurosidad ${ }^{7}$. En total, cincuenta y cinco galenos desde Imhotep y Asklepios hasta William Osler. Ocho pertenecientes a la Antigüedad Clásica, dos a la medicina islámica, dos a la Alta y Baja Edad Media, cuatro al Renacimiento, ocho al siglo

7 Sigerist, H. E. (1919), Grosse Ärzte. Fue traducida al castellano por Francisco Arasa y Manuel Scholz Rich en 1949 (Barcelona, Ediciones AVE, 310 pp.). Los traductores añadieron un apéndice en el que incluyeron, entre otras, la biografía de Santiago Ramón y Cajal. 
XVII, ocho a la Ilustración, veintiuno al siglo XIX y uno al siglo XX. De forma significativa, Sigerist dedicó su obra al médico práctico, no genial, pero que con su labor diaria ayuda a aliviar los males de la humanidad. Ese médico, olvidado por la historia ante las creaciones de los geniales, es merecedor de reconocimiento y respeto ${ }^{8}$.

El género biográfico y bio-bibliográfico cuentan en nuestro país también con una larga tradición histórica ${ }^{9}$. Baste recordar, por ejemplo, los estudios más recientes de Pedro Laín Entralgo, Luis Sánchez Granjel, Juan Riera, Agustín Albarracín, José María López Piñero o Luis García Ballester, entre otros.

Quizás la culminación en España de ese género biográfico más puro se encuentre en Grandes Médicos de Pedro Laín, redactada en 1959 y publicada en $1961^{10}$. Se trata de ocho extensas biografías dedicadas a personajes desde el Renacimiento hasta el siglo XX. Son médicos bien conocidos por Pedro Laín, a los que en otros trabajos había dedicado espléndidos análisis. Como era de esperar, Santiago Ramón y Cajal ocupa un lugar destacado. A diferencia del estudio de Sigerist, Laín incluyó notas aclaratorias. En una Nota Preliminar Laín explicitaba las razones de la publicación de esta monografía: una de orden intelectual, exponer de forma rigurosa los hechos científicos que elabora en el curso de su vida un médico creador. La segunda razón era de naturaleza moral, la obligación que tiene el historiador de mostrar el bien y la verdad de manera apasionada, pero objetiva. Hay, finalmente, una intención pedagógica. Laín pretendía que las vidas de los grandes creadores despertaran en sus lectores un ansia de emulación y de competir con ellos ${ }^{11}$. Señalaba Laín, finalmente, cinco notas que debía detener en cuenta el historiador que deseara hacer biografías con rigurosidad y objetividad:

«Hechos objetivos, ideas, modos radicales de ser (creencias, esperanzas, amores últimos) e intenciones. Sin saber con alguna precisión lo que un autor hizo, lo

8 «No son ciertamente los grandes médicos los que determinan el estado sanitario de una población, sino el ejército de los médicos prácticos que, dispersos por todo el país, se hallan al lado del enfermo» (SIGERIST, H.E. (1949), op. cit. en nota (6), p. 8)

9 Un interesante análisis del período clásico de la historia de la medicina española, concretamente, sobre Antonio Hernández Morejón, Anastasio Chinchilla, José Miguel Guardia y Luis Comenge Ferrer en: Bujosa, Homar, F. (1989), Filosofia e Historiografía médica en España. Madrid, Consejo Superior de Investigaciones Científicas [Estudios sobre la Ciencia, n. 9], 239 pp.

10 Laín Entralgo, P. (1961), Grandes Médicos. Barcelona-Madrid, Salvat Editores, S.A., 377 pp.

11 Op. cit. en nota anterior, p. VI. 
que pensó, lo que creyó, esperó y amó, lo que quiso hacer y, por añadidura, las ideas y sentimientos que en torno a sí suscitó, el cultivador de la historia quedará en ser simple, cuando no frívolo curioso del pasado» ${ }^{12}$.

El cambio del objeto de la historia hizo que el estudio de los creadores geniales fuera periclitado por otros acercamientos. La humanidad silente, las grandes epidemias, grupos marginados, como los enfermos mentales, entre otros, pasaron a primer plano y fueron la diana de los estudios historicomédicos. Frente al individuo se apostó por la recuperación de la colectividad como protagonista de la historia. La biografía se consideró como modelo de la antihistoria. No es momento de narrar con precisión estos cambios. Lo cierto es que el análisis de los genios quedaron como un objeto de estudio menor. Bien es cierto que entre nosotros algunas de las grandes figuras siguieron siendo bien atendidas ${ }^{13}$. Las razones de este desplazamiento son varias. Cierto papel jugó en la década de los setenta la aparición de los nuevos métodos historiográficos ${ }^{14}$. En el caso de Valencia, que es el que mejor conozco, este abandono se dio cuando el grupo de López Piñero apostó de manera decidida por algunas de esas nuevas técnicas, especialmente la bibliometría y el análisis semántico documental. También, López Piñero defendió el análisis prosopográfico como una nueva técnica en el estudio de grupos colectivos ${ }^{15}$. No deja de ser paradójico que el propio López Piñero editara en 1973 una monografía sobre los fundamentos doctrinales de la obra neurológica de John Hughlings Jackson ${ }^{16}$.

En los últimos años el género biográfico ha conocido en nuestro país un espectacular auge. Desde la historia general diré que en un congreso histórico internacional dedicado en parte a cuestiones metodológicas y celebrado en Santiago de Compostela en julio de 1993, en el que participaron notables historiadores, se consagró una sección a revisar el papel de la biografía en la historia ${ }^{17}$. En el

12 Ibidem, p. V.

13 Peset, J.L: Peset, M. (1975), Clásicos de la Medicina. Lombroso y la escuela positivista italiana. Madrid, Consejo Superior de Investigaciones Científicas, 745 pp.

14 Clarke, E. (Ed.) (1971), Modern Methods in the History of Medicine. London, The Athlone Press, 389 pp.

15 LÓPEZ PIÑERO, J.M. (1975), Las nuevas técnicas de investigación históricomédica. Valencia, Editorial Facta, $38 \mathrm{pp}$.

16 LÓPEZ PIÑERO, J.M. (1973), John Hughlings Jackson (1835-1911). Evolucionismo y Neurología. Madrid, Editorial Moneda y Crédito, 183 pp.

17 Colomer, F. Biografía y cambio social: la historia que estamos viviendo (pp. 67-174); Strozzi, S. Sujeto y persona en la biografía histórica. Ambos en: BARros, C. (Ed.) (1995). Historia a debate. Tomo III. Otros enfoques. Noia-A Coruña, Gráficas Sementeira. 
ámbito de la historia de la medicina y de las ciencias el punto de partida fue, sin duda, el Diccionario Histórico de la Ciencia Moderna en España, dirigido por José María López Piñero (1983) ${ }^{18}$. Ese mismo se año completaba el Diccionari Biògrafic de Metges Catalans (tres volúmenes, 1981-1983), editado por Josep M. Calbet i Camarasa y Jacint Corbella i Corbella ${ }^{19}$. Un decenio después salían a la luz el Diccionario Histórico de Médicos Vascos (1993), dirigido por Luis S. Granjel, y el Diccionario Histórico das Ciencias e das Técnicas de Galicia, coordinado por Xosé A. Fraga Vázquez y Alfonso Mato Domínguez ${ }^{20}$. Más recientemente están en marcha dos ambiciosos proyectos. Uno, promovido por la Academia de la Historia, es un diccionario biográfico español, que verá la luz a partir de 2005 y en el que se han incluido voces de científicos y médicos. El segundo, un Dictionary of Medical Biography, está siendo coordinado por W. F. Bynum, incluirá en torno a cuarenta médicos españoles y portugueses (bajo el epígrafe Iberia) y aparecerá en cinco volúmenes a partir de junio de 2005.

Como acabo de mostrar, los grandes cultivadores de esta forma de hacer historia defendieron siempre su seriedad metodológica. La rigurosidad en el análisis de las ideas, la adecuada contextualización del personaje en su época, la comprensión evolutiva del pensamiento del sujeto estudiado, entre otros elementos, han sido y son la garantía de un acercamiento riguroso. El viejo, pero totalmente actual, modo germánico de enfrentarse a la biografía de un personaje me parece que es el más adecuado y enriquecedor. En primer término conviene considerar al sujeto de estudio diacrónicamente, es decir, examinar las diferentes vidas que conforman la personalidad científica del individuo. Es lo que clásicamente se conoce como «punto de partida e influencias posteriores». A las ideas iniciales del autor se añaden, en el curso del tiempo, otras influencias-discipulares, epistolares, lecturas de obras de otros autores, relaciones personales no discipulares...- que conformarán otras vidas con otras ideas o formas de enfrentarse con los problemas y que, en ocasiones, pueden aparecer contradictorias o radicalmente diferentes a las

18 López Piñero, J.M.; Glick, Th. F.; Navarro Brotóns, V.; Portela Marco, E. (Eds.) (1983), Diccionario histórico de la ciencia moderna en España. 2 vols., Barcelona, Ed. Península.

19 Calbet I Camarasa, J.M.; Corbella I Corbella, J. (1981-1983), Diccionari Biògrafic de Metges Catalans. 3 vols., Barcelona, Editorial Rafael Dalmau.

20 Granjel, L. S. (Dir.) (1993), Diccionario Histórico de Médicos Vascos. Bilbao, Seminario de Historia de la Medicina Vasca; Fraga VÁzquez, X.A.; Mato Domínguez, A. (Coords.) (1983). Diccionario Histórico das Ciencias e das Técnicas de Galicia. Autores, 1868-1936. Sada-A Coruña, Ediciós do Castro. 
iniciales. Determinar esos diferentes planos, el inicial y los postreros, es una de las tareas más difíciles del historiador pero, no cabe duda, es profundamente satisfactoria, pues le permite conocer con claridad la mente poliédrica del autor estudiado. Como docentes es nuestra obligación mostrar a nuestros alumnos la complejidad y las contradicciones, si se dieron, en las vidas de los médicos estudiados. El estudiante debe saber que el esfuerzo intelectual es siempre muy costoso desde el punto de vista personal y que puede acompañarse de elementos disonantes que no por ello desmerecen en lo más mínimo la producción científica del biografiado. No hay que ocultar, por ejemplo, que junto a un revolucionario científico como René Théophile Hyacinthe Laënnec (1781-1826), existía otro Laënnec profundamente religioso, y escasamente rompedor en lo político. Algunos aspectos de la obra médica de Laënnec estuvieron condicionados, precisamente, por estos principios ideológicos más conservadores. Los científicos no son santos, más bien son seres humanos hijos de un tiempo, al cual se deben y del cual se empapan para crear explicaciones a los problemas que ese tiempo tiene pendientes de resolver. Pueden ser «grandes figuras» pero su adecuada comprensión exige conocer los condicionantes económicos, políticos, culturales y de todo orden que se dieron en su época. Recuérdese la actitud nacionalista y belicosa de Luis Pasteur (1822-1895) o la del International Research Council, fundado en 1919, y del que quedaron excluidos los científicos alemanes, perdedores en la Primera Guerra Mundial.

He señalado anteriormente que la biografía aséptica y edulcorada es poco interesante porque ayuda poco a pensar al estudiante. Impide la reflexión, pues todo está ya hecho y de forma óptima. Hay otras formas de acercarse a la biografía de un científico que también deben evitarse porque contienen también elementos irreales. Una de ellas es lo que se conoce como precursorismo. Es decir, estimar al objeto de estudio como un avanzado de su tiempo y creador de paradigmas altamente competitivos. Supone dar un salto en el tiempo, pues al estudiado se le hace creador de doctrinas que no tendrían lugar hasta fechas más tarde. Este modo va más allá de los predecesores, es decir, de los que abren vías intelectuales que completan los posteriores. Aquí el antes y el después se funden ucrónicamente en un mismo instante, y al mismo tiempo se restan méritos al auténtico creador de una doctrina al considerar que prácticamente otro científico precedente la había entrevisto o casi formulado en su totalidad. Obviamente que en el precursorismo suele darse una mala lectura o una interpretación excesivamente contemporánea de las fuentes en las que teóricamente se formula la doctrina posterior. Algunas veces el precursorismo está condicionado por un acercamiento excesivamente nacionalista del pasado. La caricatura de tal actitud puede encontrarse en el 
manual de historia de la medicina de Mikhail Petrovich Multanovsky (n. 1895), historiador soviético, que, prácticamente, hacía a varios médicos rusos de la época los creadores de los grandes paradigmas de la medicina moderna, los de William Harvey y Thomas Sydenham, entre otros ${ }^{21}$.

Querido señalar, finalmente otro aspecto que puede contribuir a distorsionar los resultados y transmitir una imagen equívoca al estudiante. Que me interese por una figura concreta no obliga a que, puesto que he invertido tiempo, deba concluir de forma altisonante. Una excesiva identificación del historiador con el biografiado resta objetividad. Hay que desechar el temor al fracaso. El objeto de estudio, por el hecho de ser tratado por mí, no tiene por qué ser un individuo excepcional, una gran figura. Ello presupone magnificar no sólo al estudiado, sino también el marco histórico concreto en que se desenvolvió.

\section{EL ANÁLISIS COLECTIVO: LA PROSOPOGRAFÍA}

El estudio biográfico grupal es un método ampliamente difundido en algunas ramas del saber. Es habitual, por ejemplo, en la patrística. Sin embargo, carecía de tradición en la historia de la ciencia. En 1974 S. Shapin y A. Thackray publicaban en History of Science un articulo que ha devenido un clásico de la cuestión ${ }^{22}$. Estos estudiosos señalaban que el estudio de una colectividad de científicos permite una adecuada contextualización histórico-social de ese colectivo y del momento en que realizaron su labor y ofrece una radiografía bastante fidedigna de los que ese colectivo representó en su momento. El análisis prosopográfico permite, pues, determinar lo que de común o dispar hay en un grupo de científicos y conocer en profundidad su comportamiento histórico-social. José María López Piñero fue el difusor de este método en nuestro país. El mismo lo aplicó al colectivo de científicos de la España del Renacimiento ${ }^{23}$. Otros historiadores, vinculados al grupo de Valencia, lo han utilizado en sus investigaciones. Moreno Latorre, por ejemplo, a los botánicos de la Ilustración española y José Ramón Bertomeu Sánchez a los científicos

21 Multanovski, M. P. (1967), Historia de la Medicina. La Habana, Academia de Ciencias de Cuba, 558 pp.

22 Shapin, S.; Thackray, A. (1974), Prosopography as a Research Tool in History of Science. History of Science, 22, 1-28.

23 López Piñero, J.M. (1979), Ciencia y Técnica en la sociedad española de los siglo XVI y XVII. Barcelona, Editorial Labor, pp. 47-88. 
en activo durante el gobierno de José $\mathrm{I}^{24}$. Por mi parte, en mi historia de la Facultad de Medicina de Granada he presentado un a primera ordenación de materiales referidos a los docentes de este institución, según las disciplinas que impartieron desde 1857 hasta $1976^{25}$.

La prosopografía es un método difícil y muy laborioso, si se quiere realizar con rigurosidad. El recurso a este método con fines docentes exige una gran dedicación previa, pues hay que elaborar cuidadosamente los materiales del grupo estudiado. El narrador debe, primeramente, seleccionar el grupo y, a continuación, establecer claramente las variables que se van a utilizar. El colectivo puede pertenecer a una misma institución (Academia, Universidad, Facultad, institución docente no universitaria, organismo investigador no exclusivamente docente etc..), o bien puede tratarse de miembros de una misma generación biológica (por ejemplo, la intermedia, de sabios... ), o bien compartir los mismos supuestos ideológicos (ya sea científicos o de otro orden) - como los novatores del XVII, los exiliados de la Guerra Civil Española...o ser estudiosos de los mismos problemas (tal el caso del trabajo ya citado de Moreno Latorre) o, finalmente, pertenecer a la misma escuela doctrinal. Este ultimo criterio me parece el mas etéreo y complejo de establecer, pues exige determinar primero cuales son los rasgos científicos comunes a los integrantes de tal escuela y los elementos cambiantes. No es infrecuente encontrar trabajos en los que se utiliza el concepto de escuela cuando los miembros de ese grupo lo único que compartieron fue el techo que los cobijaba, careciendo de elementos epistemológicos comunes. El artículo antes citado de Bertomeu Sánchez me parece modélico en este sentido.

La segunda dificultad consiste en delimitar claramente las variables que se van a utilizar en el análisis. En función de las características del grupo deberán establecerse esas variables. A título de ejemplo diré que en el citado trabajo de Bertomeu Sánchez se consideró la edad, el lugar de residencia, la formación, las áreas o disciplinas cultivadas, las profesiones y ocupaciones, y

24 Moreno Latorre, E. (1993). Prosopografía de los botánicos de la Ilustración española. En: Navarro Brotóns, V. (Ed.). Actes de les II Trobades d'Història de la Ciència i de la Tècnica. Penyscola, 5-8 de desembre de 1992. Barcelona, Societat Catalana d'Història de la Ciència i de la Tècnica, pp. 339-446; BERTOMEU SÁnCHEZ, J.R. (1994). Los cultivadores de la ciencia españoles y el gobierno de José I (1808-1813), Un estudio prosopográfico. Asclepio, $46(1), 125-155$.

25 OlagüE De Ros, G. (2001). Sobre sólida roca fundada: Ciento veinte años de labor docente, asistencial e investigadora en la Facultad de Medicina de Granada (1857-1976). Granada, Editorial de la Universidad, pp. 320-379 [Apéndice 7: Estudio prosopográfico de los docentes de la Facultad de Medicina de Granada, según disciplinas (1857-1976)] 
la actitud política, entre otros elementos. Otra dificultad añadida reside en las fuentes utilizadas para reconstruir la biografía de ese colectivo. Por término general no basta con las impresas (necrológicas, estudios concretos sobre el autor...) sino que se precisa recurrir a materiales de archivo, a veces no de fácil localización y consulta. Si se trata de un colectivo con una producción científica impresa, conviene finalmente relacionar sus publicaciones e intentar delimitar lo que de común o dispar hay ideológicamente en las mismas. Entiendo que es un método complejo para ser aplicado por los estudiantes en trabajos escolares. Especialmente por la gran cantidad de información que deben reunir y por la complejidad de las fuentes que deben ser utilizadas.

En la medida que el análisis prosopográfico estudia un colectivo es, en principio, un método más objetivo que el clásico estudio biográfico de un único personaje. Piénsese que el punto de partida y las influencias posteriores en este caso se extienden a cada uno de los integrantes del colectivo y a la institución misma donde realizaron su labor. Uno de los mayores atractivos de este método tiene que ver con la consideración de la ciencia como una construcción social. En este caso, es un colectivo el que crea pensamiento y que debe negociar con los demás grupos y con la sociedad la validez y pertinencia de sus hallazgos. La propia supervivencia del grupo o de la institución en la que realizan sus tareas depende, en buena parte, de su capacidad de convencimiento social de los logros obtenidos y del mantenimiento de la institución, garantía, por tanto, de continuidad de dicho grupo. Los problemas de identificación del autor con el biografiado y de transferencia afectiva es más difícil que se den, obviamente, cuando el objeto no es un sujeto concreto sino un colectivo. Pero no basta con saber que los miembros del grupo laboran en la misma institución y que comparten idénticos supuestos doctrinales. Importa también estudiar las razones de integración en el grupo; es decir, los factores - religiosos políticos o de cualquier orden - que conducen a la captación de nuevos miembros a partir de un núcleo originario.

En la prosopografía pueden darse algunos problemas comunes con la biografía individual que conviene considerar. Por un lado, pensar que el grupo objeto de estudio es un colegio invisible, es decir, un conjunto altamente creativo y rupturista en su disciplina o en las materias abordadas por el mismo, cuando de hecho no pasa de ser un colectivo con cierto interés local. La ausencia de una contextualización adecuada, pues, puede hacer caer en un falso precursorismo, aspecto que ya he comentado anteriormente. Otra deformidad que conviene obviar es el provincianismo, que en este caso surge cuando, descontextualizado el grupo, se concluye que sus logros fueron más allá de cualquier institución de su tiempo, ya sea nacional o universal. Este defecto 
puede darse de manera especial en los acercamientos mediatizados por una ideología desenfocada de corte nacionalista, en la que suele primar lo lugareño frente a una visión universal de la historia de la medicina. 